\title{
Perspective review on applications of optics in cerebral endovascular neurosurgery
}

Christopher R. Pasarikovski

Jillian Cardinell

Victor X. D. Yang 


\title{
Perspective review on applications of optics in cerebral endovascular neurosurgery
}

\author{
Christopher R. Pasarikovski, ${ }^{a, b}$ Jillian Cardinell,, ${ }^{c, d}$ and Victor X. D. Yang ${ }^{a, b, c, d, *}$ \\ aUniversity of Toronto, Division of Neurosurgery, Department of Surgery, Toronto, Ontario, Canada \\ ${ }^{b}$ Sunnybrook Health Sciences Centre, NeuroVascular Clinic, Toronto, Ontario, Canada \\ ${ }^{\mathrm{C}}$ Ryerson University, Bioengineering and Biophotonics Laboratory, Toronto, Ontario, Canada \\ dSunnybrook Health Sciences Centre, Division of Neurosurgery, Toronto, Ontario, Canada
}

\begin{abstract}
Cerebral endovascular neurosurgery has transformed the way we manage cerebrovascular disease. Several landmark trials have demonstrated the effectiveness of endovascular techniques leading to continued technological development and applications for various diseases. The utilization of optical technologies and devices is already underway in the field of endovascular neurosurgery. We discuss the contemporary paradigms, challenges, and current optical applications for the most common cerebrovascular diseases: carotid atherosclerotic disease, cerebral aneurysms, intracranial atherosclerosis, and dural arteriovenous fistulas. We also describe needs-based opportunities for future optical applications, with the goal of providing researchers a sense of where we feel optical technologies could impact the way we manage cerebral disease. $\odot$ The Authors. Published by SPIE under a Creative Commons Attribution 4.0 Unported License. Distribution or reproduction of this work in whole or in part requires full attribution of the original publication, including its DOI. [DOI: 10.1117/1.JBO.24.3.030601]
\end{abstract}

Keywords: cerebrovascular; endovascular; carotid; cerebral aneurysm; dural fistula; optics.

Paper 180684-PER received Dec. 28, 2018; accepted for publication Feb. 19, 2019; published online Mar. 26, 2019.

\section{Introduction}

Cerebral endovascular neurosurgery has transformed the way we manage cerebrovascular disease. The appeal of treating vascular diseases through minimally invasive intraluminal techniques without the need for open surgery has led to continued technological innovation and device development. ${ }^{1}$ In the era of evidence-based medicine, several landmark trials have demonstrated the effectiveness of endovascular techniques for various cerebral vascular diseases. The ISAT trial in 2005 showed that endovascular aneurysmal embolization resulted in increased independent survival at 1-year when compared to surgical clipping for ruptured brain aneurysms. ${ }^{2}$ The CREST trial in 2012 showed that carotid stenting was effective and safe when compared to surgical endarterectomy for carotid artery stenosis. ${ }^{3}$ Finally, for the management of stroke, the DAWN trial in 2018 revealed that endovascular thrombectomy was far superior to standard medical care even for patients 6 to $24 \mathrm{~h}$ after stroke onset. ${ }^{4}$ This has revolutionized stroke care for patients.

The mounting evidence behind the effectiveness of endovascular treatments and the general desire toward minimally invasive procedures will likely lead to an even greater role for endovascular management. The utilization of optical technologies and devices is already underway in the field of endovascular neurosurgery. In this paper, we highlight our perspective on current challenges faced by clinicians for various cerebrovascular diseases. Furthermore, we describe existing optical applications and opportunities for future applications.

*Address all correspondence to Victor X. D. Yang, E-mail: victor.yang@ sunnybrook.ca

\section{Carotid Atherosclerotic Disease}

\subsection{Current Paradigm and Challenges}

Stroke is one of the leading causes of morbidity and mortality in the developed world and $20 \%$ to $30 \%$ of strokes can be attributed to carotid atherosclerotic disease. ${ }^{5}$ The management algorithm for carotid atherosclerotic disease begins with patients differentiated based on the presence or absence of neurological symptoms attributed to the stenosis. Subsequently, patients are grouped based on the degree of radiographic stenosis as mild $(<50 \%)$, moderate $(50 \%$ to $69 \%)$, and severe $(70 \%$ to $99 \%)$. The landmark NASCET trial in 1991 showed that surgical intervention is highly beneficial for symptomatic patients with moderate and high-grade stenosis. ${ }^{6}$ The results of the NASCET trial remain influential today, and the current American Heart Association/American Stroke Association recommends carotid revascularization for symptomatic patients with moderate and severe stenosis. ${ }^{7}$

However, there is mounting evidence that the degree of radiographic stenosis fails to identify those patients with vulnerable carotid plaques. Several authors have reported carotid plaque morphological features, such as degree of lipid content, thickness of the fibrous cap, calcification, and intraplaque hemorrhage could all indicate plaque instability, and thus higher risk of stroke (Fig. 1). ${ }^{8-10}$ Identifying these high-risk morphological features would change the way we treat patients with carotid stenosis. Current noninvasive imaging techniques include MRI, CT, and ultrasound. However, no modality has proven reliable in identifying vulnerable plaques in relation to future stroke development. ${ }^{11}$

Furthermore, once the decision is made to treat the carotid stenosis, physicians have two options: carotid endarterectomy and carotid artery stenting (CAS). The CREST trial showed that carotid stenting was associated with increased risk of 

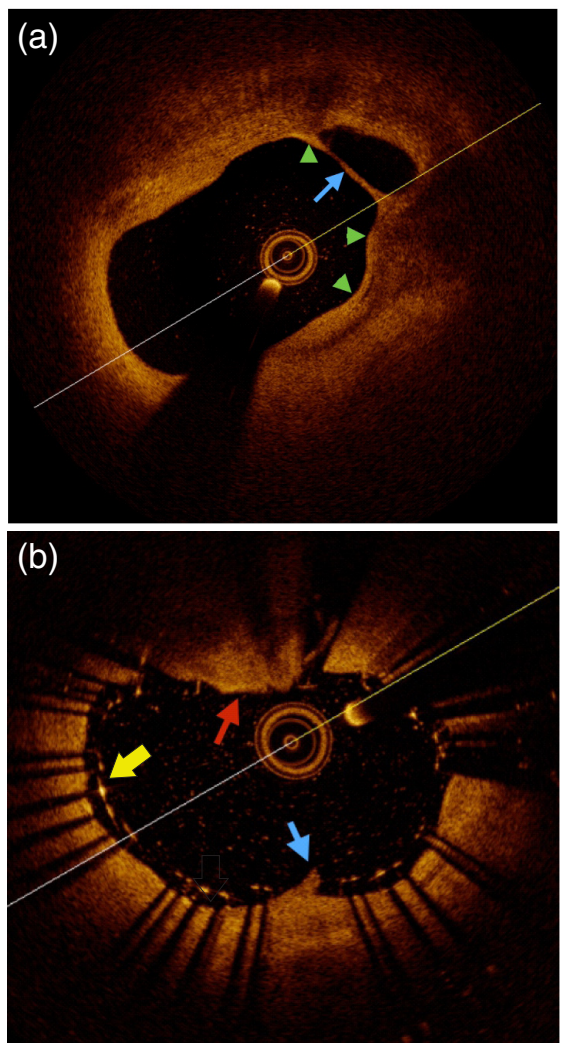

Fig. 1 OCT imaging of carotid plaque before and after stenting. (a) Example of thin fibrous cap (green arrows) over a necrotic core (blue arrow) before stenting. These are both concerning plaque features increasing the future risk of stroke. (b) Poststenting example of stent-strut malapposition (yellow arrow), plaque prolapse through the stent (blue arrow), and thrombus over the stent (red arrow). These features are high-risk for stoke development.

perioperative stroke compared to endarterectomy. ${ }^{3}$ Authors have hypothesized that plaque prolapse through the stent-struts as a major cause of perioperative stroke. ${ }^{12}$ Currently, imaging modalities are unable to detect this prolapse after stent placement.

\subsection{Current Optical Applications}

With respect to noninvasive carotid stenosis imaging, authors have described imaging the plaques with laser ultrasound. ${ }^{13}$ They report improved vessel wall spatial resolution compared to $\mathrm{CT}$ and the ability to detect calcification. However, the high acoustic contrast factor between calcium and surrounding tissue results in shadowing beyond the calcified plaques, leading to an inability to image beyond the lesion. With respect to invasive imaging, the United States Food and Drug Administration (FDA) approved the use of optical coherence tomography (OCT) for intravascular imaging in 2010. The commercially available OCT catheter is approved for coronary imaging and not carotid imaging. Given et al. were the first group to describe the use of OCT for carotid plaques using saline without proximal balloon occlusion. ${ }^{14}$ Since then, several authors have studied carotid plaque morphology and composition. ${ }^{12,14-22}$ Others have described the use of OCT for detecting plaque prolapse and stent-strut malapposition during CAS (Fig. 1). ${ }^{12,15,19-25}$

Several limitations of carotid atherosclerotic OCT imaging exist. Clinicians have described satisfactory imaging without proximal balloon occlusion, though it is our institutional experience that the highest quality images are obtained with proximal occlusion. The occlusion temporarily blocks blood flow to the brain and consequently has potential for ischemic injury. We have not encountered complications in our experience given the short ( $6 \mathrm{~s}$ ) acquisition time. Furthermore, the FDA-approved device is designed for imaging vessels 2 to $3 \mathrm{~mm}$ in diameter and the carotid artery is 5 to $8 \mathrm{~mm}$ in diameter, leading to an inability to image the entire vessel in certain patients. Lesions such as calcium or red thrombus also cause shadowing.

Intraluminal angioscopic imaging for carotid atherosclerotic disease has also been described with excellent visualization of the plaques. ${ }^{26}$ The major disadvantage for this technology is the requirement of long-duration proximal balloon occlusion during acquisition. Newer laser-based angioscopes have showed promise discerning the different phases of atherosclerotic plaque evolution in animal models. ${ }^{27}$

\subsection{Future Directions}

The calculation of radiographic degree of stenosis to determine which patients should be treated for carotid disease is likely rudimentary. Ideally, a noninvasive technology would exist to image a carotid plaque for features that would predict future risk of stroke. Shindo et al. ${ }^{28}$ in 2015 showed that carotid plaque fibrous cap thickness $<130 \mu \mathrm{m}$ was associated with increased risk of rupture, and thus any new technology would need superb spatial resolution. The device would also need to be able to differentiate a lipid core, calcium deposits, intraplaque rupture, and thrombus on the surface of the plaque. A clinical trial to assess the predictive value of a noninvasive optical device able to detect vulnerable plaque features would be very appealing to clinicians given the massive burden of stroke.

A device to detect plaque prolapse through the struts of a stent during CAS would also be an extremely valuable tool for clinicians. As described above, we know from the CREST trial that periprocedural stroke was higher in the CAS group and some authors have hypothesized that plaque prolapse through the stent could be the cause. ${ }^{29}$ The device must have the spatial resolution adequate to identify plaque on the micrometer scale and be able to image a vessel with $\sim 5$ to $8 \mathrm{~mm}$ diameter. The detection of plaque prolapse would be done after stent placement by imaging over the stent mesh. If thrombus/prolapse were detected, periprocedural anticoagulation could be used to decrease the risk of thromboembolism.

What we envision is a not a new standalone optical technology to detect plaque prolapse, rather a combination of several different technologies into an all-purpose stenting device with imaging capability (Fig. 2). The device would ideally be comprised of a distal embolization protection system, a dual-lumen transparent angioplasty balloon filled with saline that optical imaging can be acquired through the balloon, a stent, and a proximal balloon for occlusion during stent deployment. This would save a significant amount of time during the procedure, as several catheters would not need to be exchanged during optical imaging.

\section{Cerebral Aneurysm}

\subsection{Current Paradigm and Challenges}

The prevalence of intracranial aneurysms in the population is $\sim 3.2 \%{ }^{30}$ Cerebral catheter angiography remains the gold- 


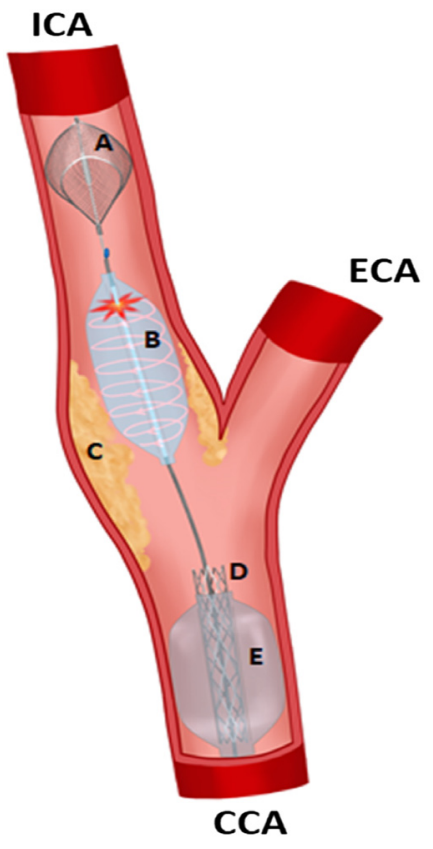

Fig. 2 Carotid stenosis treated using a novel carotid stenting device with integrated optical technology. (A) Distal embolization protection system that captures all possible blood-clots during the procedure to prevent strokes. (B) Saline filled angioplasty balloon used to first widen the narrowed artery. An optical imaging catheter images the plaque through the dual lumen balloon after angioplasty. (C) The carotid atherosclerotic disease that is responsible for artery narrowing and producing blood-clots, which cause strokes in the brain. (D) The carotid stent, which will be placed to keep the narrowed artery open. (E) Proximal dual lumen balloon that will be inflated at various times during the procedure for proximal blood-flow arrest to prevent bloodclots from traveling to the brain. ICA, Internal carotid artery; ECA, external carotid artery; CCA, common carotid artery.

standard in diagnosing cerebral aneurysms. The anatomic location, shape (saccular or fusiform), and size can all be determined with great accuracy. When dealing with unruptured aneurysms, this information along with patient-specific characteristics allows for an estimation of the future risk of rupture for the aneurysm. This information is paramount when deciding whether to treat an aneurysm or opt for observational management. ${ }^{31}$ There is increasing evidence to suggest that aneurysmal wall thickness and histologic composition are heterogeneous, and these differences may play a role in aneurysmal pathogenesis and risk of rupture. ${ }^{32}$ Current imaging modalities, limited by spatial resolution, cannot provide information on aneurysm wall characteristics.

With respect to management, there has been a revolution in the way we treat aneurysms after the ISAT trial in 2005 . $^{2}$ Most ruptured aneurysms in the United States are now are treated endovascularly. ${ }^{33}$ Different techniques for endovascular aneurysmal embolization are utilized including: coiling, stentassisted coiling, and flow-diverting stents (Fig. 3). Although endovascular treatment has improved patient care, aneurysm recurrence rates due to coil compaction/recanalization can approach $20 \% .{ }^{34}$ Similar aneurysmal recurrence rates have been reported with the use of flow-diverting stents. ${ }^{35}$ The exact mechanism behind recurrence remains elusive and some authors have suggested poor stent-strut apposition against the vessel wall at the aneurysms neck as a potential cause. ${ }^{36}$ Along with the possibility of rerupture of the aneurysm, institutions have
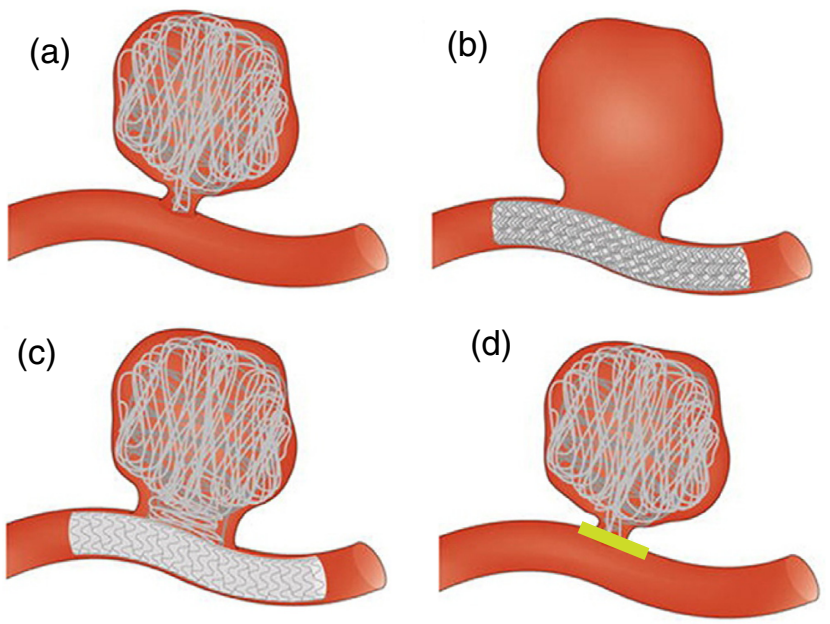

Fig. 3 Adapted from Ref. 37. Various endovascular techniques utilized for cerebral aneurysm embolization. (a) Coiling of cerebral aneurysm. The coils fill the aneurysm and cause flow stasis and subsequent thrombosis. (b) Flow-diverting stent of cerebral aneurysm. Flow-arrest in the aneurysm will lead to thrombosis of the aneurysm. (c) Stent-assisted coiling of cerebral aneurysm utilizes both stents and coils for complex aneurysms. (d) Example of a previously coiled aneurysm with neoendothelialization (yellow-line) excluding the aneurysm from the systemic circulation. If an optical device could show an aneurysm has healed, we could eliminate costly follow-up imaging.

posttreatment imaging protocols to monitor for aneurysmal recurrence which are very time consuming and very costly.

\subsection{Current Optical Applications}

The first reported in vivo intraluminal imaging of the deployment of aneurysm coils and flow-diverting stents was by done by McVeigh et al. ${ }^{38}$ They used an angioscope for direct visualization of the coils and stents in three swine experiments. The angioscope generated superb images not previously demonstrated (Fig. 4). Mathews et al. ${ }^{39}$ were the first to use a custom-built OCT device to image the cavernous internal carotid artery in three patients who previously underwent aneurysmal coiling. They showed that the internal elastic lamina and external elastic lamina of the vessel wall could be imaged intracranially with excellent resolution. Guerrero et al. ${ }^{40}$ examined neoendothelialization over a flow-diverting stent placed 8-weeks prior using a commercially available OCT catheter in the posterior circulation and found that there was concentric neoendothelialization over the stent, except in areas where the stent was not in contact with the vessel wall.

\subsection{Future Applications}

Our current knowledge of rupture risk for intracranial aneurysms is limited due to a poor understanding of the natural history. This is likely due in part to our inability to image the aneurysm wall and understand the histologic composition. Knowledge of aneurysm wall composition could have immense diagnostic potential in evaluating rupture risk for patients, and thus guide the clinical decision making process. The benefit solely from invasive aneurysmal wall imaging with an optical technology would unlikely outweigh the risks. However, the device could be utilized during diagnostic cerebral angiography and as an adjunct before/after aneurysmal treatment to provide information regarding wall histologic features. 


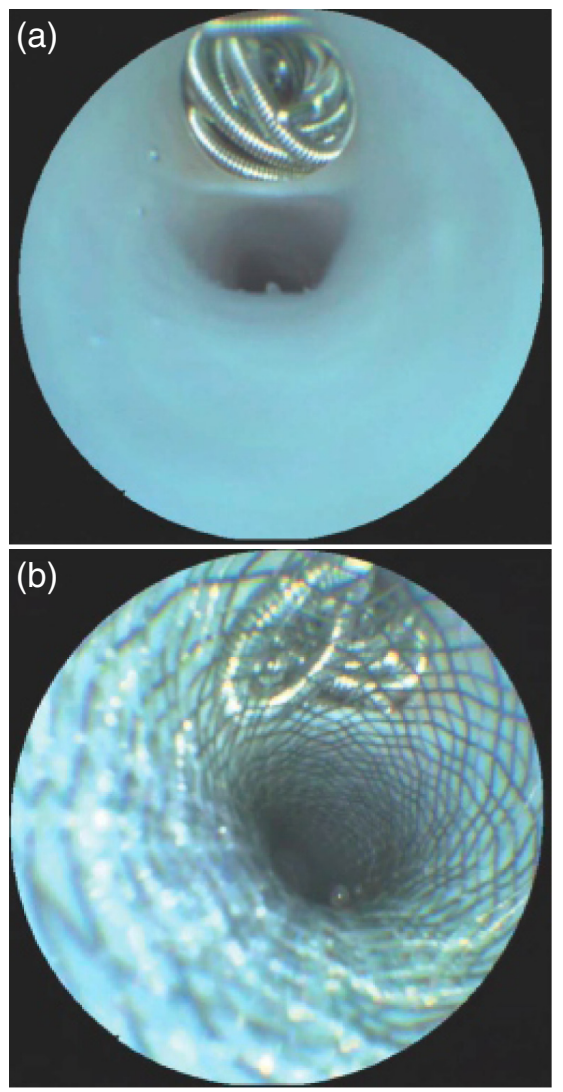

Fig. 4 Adapted from Ref. 38. Angioscope images of aneurysm coiling and stent placement. (a) Angioscope direct visualization of coil placement into the vessel. (b) Direct visualization of stent-assisted coiling of vessel. The coils can be seen through the stent mesh. Satisfactory apposition of the stent-struts against the vessel wall can also be visualized.

A device that can be navigated intracranially and confirm acceptable stent-strut apposition against the vessel wall during aneurysm embolization would be extremely valuable as an adjunct during aneurysm embolization. The lack of neoendothelialization over a stent is thought to be a risk factor for aneurysm recurrence. Intraprocedural knowledge of stent malapposition could guide the clinician to reposition the stent. Furthermore, evidence of complete neoendothelialization of stents could also help direct management with respect to duration of antiplatelet medications. Blood-thinners are needed after stent deployment because stents are thrombogenic. A stent that is completely excluded from the vessel lumen due to neoendothelialization would of course be at a decreased risk for thromboembolic events, and patients may not require blood-thinning medication. Knowledge on whether an aneurysm has been excluded from the cerebral vasculature due to complete aneurysm neck endothelialization after stenting/coiling could also eliminate the need for routine follow-up imaging looking for recurrence (Fig. 3). The above concepts all fall under the umbrella of "aneurysmal healing" after treatment, and any technology able to assess aneurysmal healing would be a powerful tool.

There are very few reported cases of endovascular imaging of intracranial vessels. One of the major obstacles for designing any device for intracranial endovascular imaging is navigating the tortuous cerebral vessels. The carotid siphon is particularly challenging. There have been several case reports of an inability to navigate catheters beyond the siphon and having the hardware fail during navigation. In our own institutional experience, we can confirm the difficulty of navigating intracranially. Models do exist to replicate the tortuous anatomy of the carotid siphon for future device testing. ${ }^{41}$

\section{Intracranial Atherosclerosis}

\subsection{Current Paradigm and Challenges}

Intracranial atherosclerosis (ICAS) is also an important etiology of stroke, especially in Asian populations. ${ }^{42}$ The landmark WASID trial in 2005 studied medical management for patients with symptomatic large vessel ICAS of $50 \%$ to $99 \% .{ }^{43}$ They compared Warfarin with Aspirin and found the primary endpoint of stroke, brain hemorrhage, or death from nonvascular causes occurred in $22 \%$ of patients in both groups. These poor outcomes lead the SAMMPRIS trial investigators in 2011 to compare medical management with intracranial stenting for patients with symptomatic large vessel ICAS of $70 \%$ to $99 \% .^{44}$ The trial was halted after interim analysis showed patients undergoing stenting had a 30-day perioperative stroke rate of $15 \%$. The majority of strokes in the SAMMPRIS trial occurred due to adjacent arterial perforator occlusion. One proposed mechanism of perforator infarct during intracranial stenting is the displacement of unstable or complex plaques into adjacent perforating vessels. ${ }^{45}$

\subsection{Current Optical Applications}

There have been no optical technologies directly utilized in the diagnosis or treatment of ICAS. Alles et al. ${ }^{46}$ were the first to image ex vivo aortic tissue with an optically transmitted acoustic beam. They demonstrated side-branch blood vessels with great accuracy (Fig. 5).

\subsection{Future Applications}

As described above, the majority of strokes in the SAMMPRIS trial occurred due to adjacent arterial perforator occlusion. The ability to directly visualize perforators before stent placement would greatly improve the safety of intracranial stenting. The perforators coming off the basilar artery or middle cerebral artery range between 80 and $1400 \mu \mathrm{m}$ in size and thus the device to image these vessels would need excellent spatial resolution. Furthermore, the detection of vulnerable plaque features could be a warning sign for the interventionalist and help decrease the perioperative stroke risk. If the risk of perforator occlusion were deemed high, the decision could be made not to place a stent. In other circumstances, different stent types (open/closed cell) could be used depending on plaque morphology. Future trials must select the appropriate patients for intervention, and a device that can identify both vulnerable plaque features and adjacent perforating vessels would certainly help identify these patients.

\section{Concept}

Arteriovenous fistulas are abnormal connections between arteries and veins. In the brain, dural arteriovenous fistulas (dAVF) acquired vascular malformations with arterial feeders from extracranial arteries. The venous system is a low-pressure system, and arterial pressure can cause symptoms varying from tinnitus (ringing in the ears) to fatal ruptures. With the evolution of endovascular neurosurgery, the first-line treatment option for 


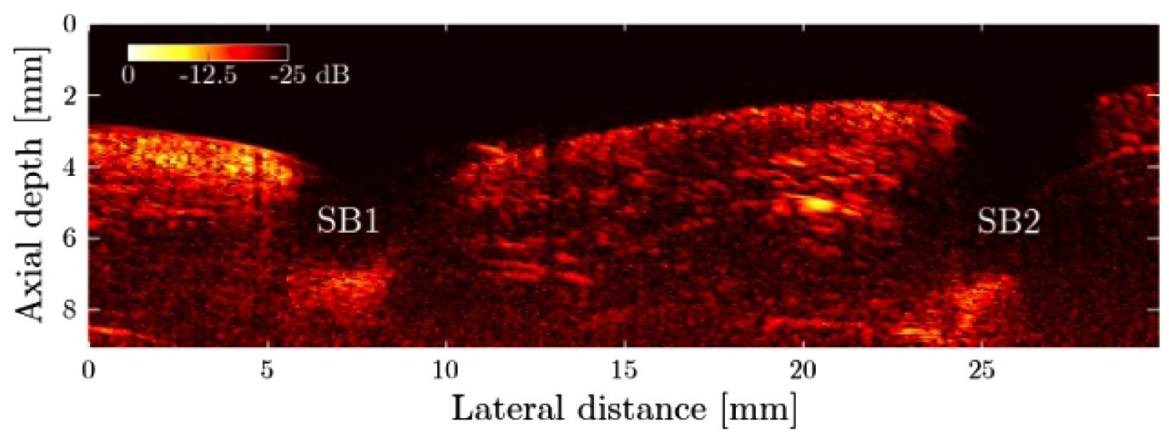

Fig. 5 Adapted from Ref. 46. Imaging of side branch 1 (SB1) and side branch 2 (SB2) coming off the aorta using all-optical ultrasound without reconstruction. The detection of side branches during intracranial stenting for atherosclerotic disease could decrease the risk of stroke during stent placement.

(a)

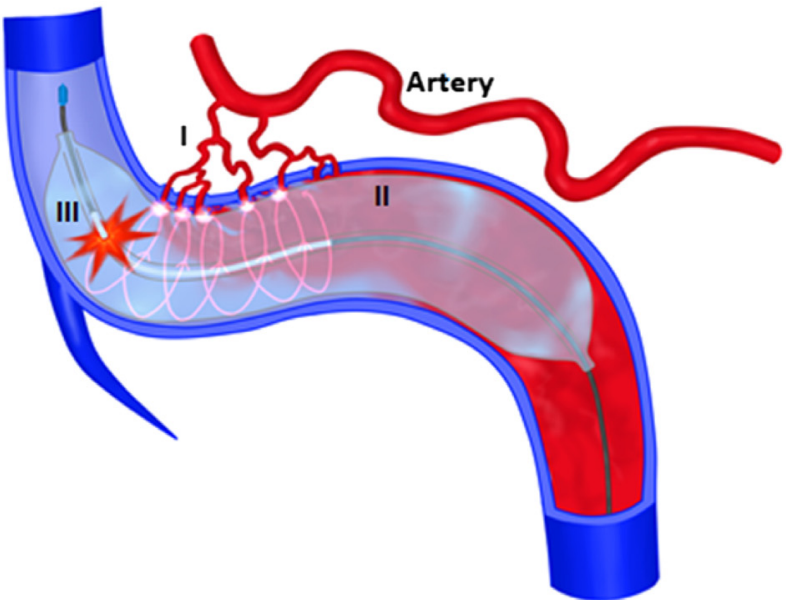

(b)

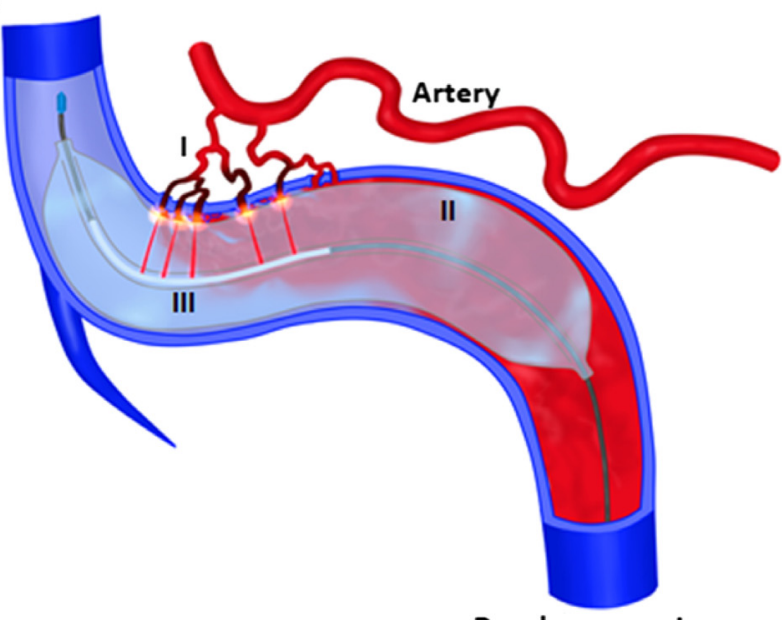

Dural venous sinus

Fig. 6 dAVF imaged and treated using optical technologies. (a) The device is navigated into the dural venous sinus and through a transparent dual-lumen balloon (II); optical imaging is undertaken (III) to identify the exact spatial position of arterial feeders (I) into the dural venous sinus. (b) Laser ablation of the arterial feeders is accomplished under image guidance through the saline-filled balloon. The arterial feeders (I) previously identified now undergo image-guided laser ablation (III) through the transparent dual lumen balloon (II). This device could eliminate the need for current glue-embolization techniques which are extremely costly and often require several treatment sessions. most dAVF is now endovascular embolization. ${ }^{47}$ Trans-arterial embolization techniques using glue are often very expensive, in the range of $\$ 20,000$ per procedure, and may require multiple procedures.

We envision an optical device that can both image the arterial feeders to the fistula and ablate these arteries using a laser (Fig. 6). First, the device would be navigated transvenously to the site of the dural AVF. Next, through a transparent dual-lumen balloon, optical imaging is undertaken to identify the exact location of arterial feeders into the dural sinus. With the knowledge of the exact spatial position of the feeding arteries, laser ablation of the feeders is completed under image guidance through the saline-filled balloon. This could eliminate the need for expensive glue embolization material and multiple treatment sessions with the use of a single device.

\section{Conclusion}

Endovascular neurosurgical management of cerebrovascular disease will continue to expand. Optical devices have begun to play a role in diagnosis and treatment. We believe there remains significant opportunity for improvement of current optical systems and techniques, along with the development of devices to treat these complex diseases.

\section{Disclosures}

The other authors declare that they have no competing interests to disclose.

\section{Acknowledgments}

This research did not receive any specific grant from funding agencies in the public, commercial, or not-for-profit sectors. Research ethics board approval was not required for this systematic review of the literature.

\section{References}

1. L. N. Hopkins and R. D. Ecker, "Cerebral endovascular neurosurgery," Neurosurgery 62, 1483-1501, discussion 501-2 (2008).

2. A. J. Molyneux et al., "International subarachnoid aneurysm trial (ISAT) of neurosurgical clipping versus endovascular coiling in 2143 patients with ruptured intracranial aneurysms: a randomized comparison of effects on survival, dependency, seizures, rebleeding, subgroups, and aneurysm occlusion," Lancet 366(9488), 809-817 (2005).

3. T. G. Brott et al., "Stenting versus endarterectomy for treatment of carotid-artery stenosis," N. Engl. J. Med. 363(1), 11-23 (2010). 
4. R. G. Nogueira et al., "Thrombectomy 6 to 24 hours after stroke with a mismatch between deficit and infarct," N. Engl. J. Med. 378(1), 11-21 (2018).

5. L. B. Goldstein et al., "Guidelines for the primary prevention of stroke: a guideline for healthcare professionals from the American Heart Association/American Stroke Association," Stroke 42(2), 517-584 (2011).

6. North American Symptomatic Carotid Endarterectomy Trial Collaborators, "Beneficial effect of carotid endarterectomy in symptomatic patients with high-grade carotid stenosis," N. Engl. J. Med. 325(7), 445-453 (1991).

7. W. N. Kernan et al., "Guidelines for the prevention of stroke in patients with stroke and transient ischemic attack: a guideline for healthcare professionals from the American Heart Association/American Stroke Association," Stroke 45(7), 2160-2236 (2014).

8. N. Takaya et al., "Association between carotid plaque characteristics and subsequent ischemic cerebrovascular events: a prospective assessment with MRI-initial results," Stroke 37(3), 818-823 (2006).

9. T. Saam et al., "The vulnerable, or high-risk, atherosclerotic plaque: noninvasive MR imaging for characterization and assessment," Radiology 244(1), 64-77 (2007).

10. G. M. Biasi et al., "Carotid plaque echolucency increases the risk of stroke in carotid stenting: the Imaging in Carotid Angioplasty and Risk of Stroke (ICAROS) study," Circulation 110(6), 756-762 (2004).

11. A. Huibers et al., "Non-invasive carotid artery imaging to identify the vulnerable plaque: current status and future goals," Eur. J. Vasc. Endovasc. Surg. 50(5), 563-572 (2015).

12. G. de Donato et al., "Optical coherence tomography after carotid stenting: rate of stent malapposition, plaque prolapse and fibrous cap rupture according to stent design," Eur. J. Vasc. Endovasc. Surg. 45(6), 579-587 (2013).

13. J. L. Johnson et al., "All-optical extravascular laser-ultrasound and photoacoustic imaging of calcified atherosclerotic plaque in excised carotid artery," Photoacoustics 9, 62-72 (2018).

14. C. A. Given, II, et al., "Frequency-domain optical coherence tomography assessment of human carotid atherosclerosis using saline flush for blood clearance without balloon occlusion," AJNR Am. J. Neuroradiol. 34(7), 1414-1418 (2013)

15. S. Dohad et al., "Optical coherence tomography guided carotid artery stent procedure: technique and potential applications," Catheter Cardiovasc. Interv. 91(3), 521-530 (2018).

16. C. A. Given, II, et al., "Optical coherence tomography of the intracranial vasculature and Wingspan stent in a patient," J. Neurointerv. Surg. 7(6), e22 (2015).

17. M. R. Jones et al., "Intravascular frequency-domain optical coherence tomography assessment of carotid artery disease in symptomatic and asymptomatic patients," JACC Cardiovasc. Interv. 7(6), 674-684 (2014)

18. I. J. Patel et al., "Novel use of optical coherence tomography," J. Neurointerv. Surg. 5(6), e41 (2013).

19. M. Tsujimoto et al., "Optimal platelet function test for in-stent tissue protrusion following carotid artery stenting," J. Int. Med. Res. 46(5), 1866-1875 (2018).

20. T. Umemoto et al., "Optical coherence tomography assessment of newgeneration mesh-covered stents after carotid stenting," EuroIntervention 13(11), 1347-1354 (2017).

21. K. Yamada et al., "Potential of new-generation double-layer micromesh stent for carotid artery stenting in patients with unstable plaque: a preliminary result using OFDI analysis," World Neurosurg. 105, 321-326 (2017).

22. S. Yoshimura et al., "Demonstration of intraluminal thrombus in the carotid artery by optical coherence tomography: technical case report," Neurosurgery 67, onsE305 (2010).

23. Endarterectomy for Asymptomatic Carotid Artery Stenosis, "Executive committee for the asymptomatic carotid atherosclerosis study," J. Am. Med. Assoc. 273(18), 1421-1428 (1995).

24. M. R. Jones et al., "Intravascular frequency-domain optical coherence tomography assessment of atherosclerosis and stent-vessel interactions in human carotid arteries," AJNR Am. J. Neuroradiol. 33(8), 1494-1501 (2012).

25. K. Harada, S. Oshikata, and M. Kajihara, "Optical coherence tomography evaluation of tissue prolapse after carotid artery stenting using closed cell design stents for unstable plaque," J. Neurointerv. Surg. 10(3), 229-234 (2018).

26. H. Tanemura et al., "Angioscopic observation during carotid angioplasty with stent placement," Am. J. Neuroradiol. 26(8), 1943-1948 (2005).

27. L. E. Savastano et al., "Multimodal laser-based angioscopy for structural, chemical and biological imaging of atherosclerosis," Nat. Biomed. Eng. 1, 0023 (2017).

28. S. Shindo et al., "Morphologic features of carotid plaque rupture assessed by optical coherence tomography," Am. J. Neuroradiol. 36(11), 2140-2146 (2015).

29. A. Abizaid and D. Chamie, "A journey into the carotid artery microenvironment in high resolution: challenging the stenosis-symptoms paradigm," JACC Cardiovasc. Interv. 7(6), 685-687 (2014).

30. M. W. Vernooij et al., "Incidental findings on brain MRI in the general population," N. Engl. J. Med. 357(18), 1821-1828 (2007).

31. J. P. Greving et al., "Development of the PHASES score for prediction of risk of rupture of intracranial aneurysms: a pooled analysis of six prospective cohort studies," Lancet Neurol. 13(1), 59-66 (2014).

32. L. M. Kadasi, W. C. Dent, and A. M. Malek, "Cerebral aneurysm wall thickness analysis using intraoperative microscopy: effect of size and gender on thin translucent regions," J. Neurointerv. Surg. 5(3), 201-206 (2013)

33. G. A. Smith et al., "Geographic variation and regional trends in adoption of endovascular techniques for cerebral aneurysms," J. Neurosurg. 114(6), 1768-1777 (2011)

34. S. P. Ferns et al., "Coiling of intracranial aneurysms: a systematic review on initial occlusion and reopening and retreatment rates," Stroke 40(8), e523-e529 (2009).

35. J. Raymond et al., "Flow diversion in the treatment of aneurysms: a randomized care trial and registry," J. Neurosurg. 127(3), 454-462 (2017)

36. R. M. King et al., "Communicating malapposition of flow diverters assessed with optical coherence tomography correlates with delayed aneurysm occlusion," J. Neurointerv. Surg. 10(7), 693-697 (2018).

37. M. N. Ngoepe et al., "Thrombosis in cerebral aneurysms and the computational modeling thereof: a review," Front. Physiol. 9, 306 (2018).

38. P. Z. McVeigh et al., "High-resolution angioscopic imaging during endovascular neurosurgery," Neurosurgery 75(2), 171-180, discussion 9-80 (2014).

39. M. S. Mathews et al., "Neuroendovascular optical coherence tomography imaging and histological analysis," Neurosurgery 69(2), 430-439 (2011).

40. B. P. Guerrero et al., "First human evaluation of endothelial healing after a pipeline flex embolization device with shield technology implanted in posterior circulation using optical coherence tomography," Neurointervention 13(2), 129-132 (2018).

41. H. Q. Tan et al., "Surgical construction of a novel simulated carotid siphon in dogs," J. Neurosurg. 109(6), 1173-1178 (2008).

42. P. B. Gorelick et al., "Large artery intracranial occlusive disease: a large worldwide burden but a relatively neglected frontier," Stroke 39(8), 2396-2399 (2008)

43. M. I. Chimowitz et al., "Comparison of warfarin and aspirin for symptomatic intracranial arterial stenosis," N. Engl. J. Med. 352(13), 1305-1316 (2005)

44. M. I. Chimowitz et al., "Stenting versus aggressive medical therapy for intracranial arterial stenosis," N. Engl. J. Med. 365(11), 993-1003 (2011).

45. C. A. Given, II, et al., "Optical coherence tomography of the intracranial vasculature and Wingspan stent in a patient," BMJ Case Rep. 2014 (2014).

46. E. J. Alles et al., "Pencil beam all-optical ultrasound imaging," Biomed. Opt. Express 7(9), 3696-3704 (2016).

47. E. Piechowiak et al., "Endovascular treatment of dural arteriovenous fistulas of the transverse and sigmoid sinuses using transarterial balloon-assisted embolization combined with transvenous balloon protection of the venous sinus," AJNR Am. J. Neuroradiol. 38(10), 1984-1989 (2017)

Christopher R. Pasarikovski obtained his honors BSc degree in medical physics at the University of Western Ontario before gaining acceptance into medical school. He completed medical school in 2015 at Western, and is currently a fourth-year neurosurgical resident 
at the University of Toronto. Following his third year, Chris entered the surgeon-scientist training program and is currently enrolled as a $\mathrm{PhD}$ candidate in the Institute of Medical Science. His research focus is the application of optical coherence tomography imaging in cerebrovascular disease.

Jillian Cardinell is an undergraduate student at Ryerson University studying biomedical engineering. Her research focus is on the analysis of retinal and dermal optical coherence tomography images for diagnostics and characterization at Sunnybrook Health Sciences Center under the supervision of Dr. Yang. She has also participated in augmented reality studies for surgical navigation. She combines her artistic abilities with her cerebrovascular technical knowledge to generate descriptive diagrams for publications.

Victor X. D. Yang received his honors BASc degree in engineering science from the University of Toronto in 1997. He completed a master's in electrical and computer engineering in 1998, then entered the University of Toronto MD-PhD program in the Department of Medical Biophysics, completed in 2004. Currently, he is an associate professor in the Division of Neurosurgery, University of Toronto, and Department of Electrical and Computer Engineering, Ryerson University, holding the Canada Research Chair (CRC) in Bioengineering and Biophotonics. 